\title{
Fine-Scale Cavitation of Ionospheric Plasma Caused by Inertial Alfvén Wave Ponderomotive Force
}

\author{
P. M. Bellan \\ California Institute of Technology, Pasadena, California 91125 \\ K. Stasiewicz \\ Swedish Institute of Space Physics, Uppsala Division, S-755 91 Uppsala, Sweden
}

(Received 25 September 1997)

\begin{abstract}
Deep, very narrow magnetic-field-aligned density depletions were observed by the Freja spacecraft during auroral oval crossings. These cavities have perpendicular width of the order of the electron skin depth $c / \omega_{p e}$ and are associated with low-frequency electromagnetic perturbations and with discrete auroral structures. We demonstrate here that these cavities are likely produced by the ponderomotive force of inertial Alfvén waves. [S0031-9007(98)05866-9]
\end{abstract}

PACS numbers: 94.20.Yx, 52.35.Bj, 95.30.Qd

Surprisingly deep and sharp plasma density cavities have been measured in auroral regions at altitudes of $\sim 1500 \mathrm{~km}$ by the Freja spacecraft [1]. These cavities have widths of order the electron skin depth $c / \omega_{p e}$ and density depletions sometimes exceeding $50 \%$. Their cause, a subject of considerable discussion, has been variously suggested as being solitary kinetic Alfvén waves [2-5], nonlinear vortices [6], or Alfvén cones [7].

The purpose of this Letter is to show that these density cavities result from the ponderomotive force created by the large field-aligned oscillating current [7] of an inertial Alfvén shear mode. Unlike previous models which tended to ignore dynamics parallel to the equilibrium magnetic field, we find that parallel electric fields and the resulting parallel electron motion (i.e., electron inertia effects) are of fundamental importance. We demonstrate in this Letter that very weak oscillating parallel electric fields associated with the inertial Alfvén shear mode can lead to substantial density depletions in magnetized plasma; this behavior is analogous in some ways to the density depletion associated with large amplitude lower hybrid resonance cones [8] and has important implications for the creation of striated cavities and other nonlinear phenomena in low $\beta$ space plasmas.

The inertial Alfvén shear mode is a cold-plasma wave which exists when the plasma $\beta$ is smaller than the mass ratio $m_{e} / m_{i}$ (or, equivalently, the Alfvén velocity $v_{A}$ is larger than the electron thermal velocity $v_{T e}$ ). This mode involves finite parallel electric field and finite electron mass (in contrast to ideal MHD where both these quantities are assumed to be vanishingly small). The parallel acceleration of finite-mass electrons explicitly determines the parallel current (this physics is neglected in ideal MHD). Superposition of the $\mathbf{k}$ spectrum generated by a localized source excites resonance cones $[9,10]$, and it has recently been shown that small-scale auroral shear Alfvén signals are consistent with resonance cone excitation by a distant, pulsed, localized source [11].
We begin by presenting in Fig. 1(a) typical Freja measurements of the transverse magnetic field $B_{\perp}$; these perturbations have been interpreted as being due to inertial Alfvén resonance cones [7]. Figure 1(a) shows that the magnetic structure is irregular and occasionally has sharp gradients. Each time increment of $1 \mathrm{sec}$ in Fig. 1 corresponds to Freja traveling a distance of $6.7 \mathrm{~km}$ perpendicular to the equilibrium magnetic field. Figure 1(b) shows measurements of the corresponding electron density. The sharp density cavities evident in Fig. 1(b) are correlated with the magnetic field gradients in Fig. 1(a). These measurements were made in auroral regions at an altitude of $1500 \mathrm{~km}$ where $T_{e}=0.5-1 \mathrm{eV}, B_{0}=2.6 \times$ $10^{4} \mathrm{nT}$, and the oxygen content of the plasma was typically $50 \%-75 \%$ with the balance being mainly hydrogen. Thus, $\beta \ll m_{e} / m_{i}$ so that the shear Alfvén modes are

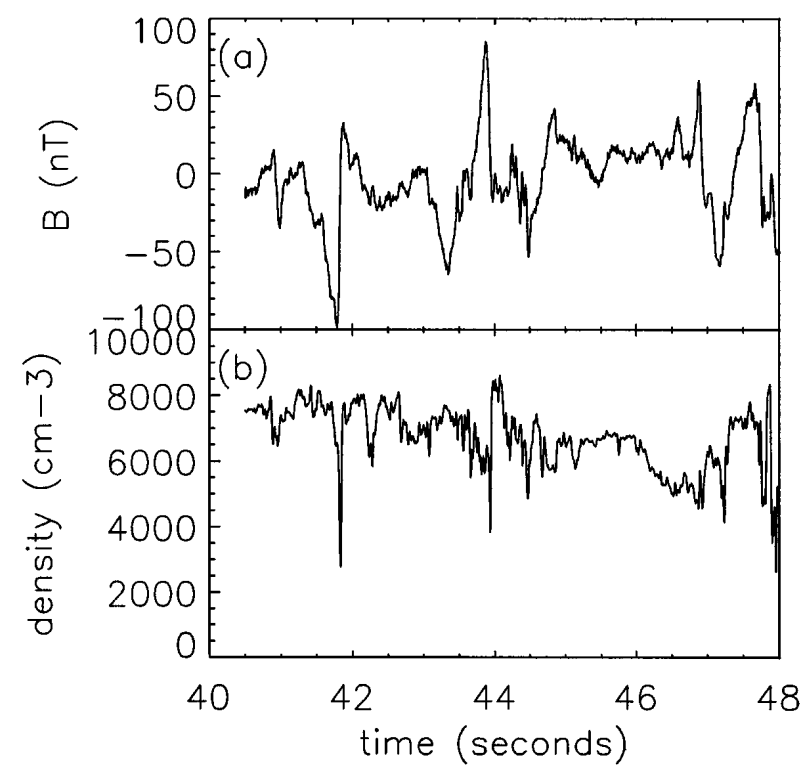

FIG. 1. (a) Perpendicular magnetic field measured by Freja on orbit 2396 at $1503 \mathrm{UT}$ (the data rate is 128 samples/sec). (b) Corresponding electron density. 
inertial modes. The electron-ion collision frequency for $T_{e}=1 \mathrm{eV}, n=8 \times 10^{3} \mathrm{~cm}^{-3}$ is $v_{e i} \sim 0.4 \mathrm{~s}^{-1}$, and so for wave frequencies $\omega / 2 \pi \sim 1 \mathrm{~Hz}$, the waves may be considered as being collisionless.

We now outline a model which relates the density cavities in Fig. 1(b) to the magnetic field gradients in Fig. 1(a). Bhattacharyya et al. [12] showed that the ponderomotive force on a charged particle in a uniform magnetic field $\mathbf{B}_{0}=B_{0} \hat{z}$ due to an arbitrary electromagnetic wave is conservative and has the form

$$
\mathbf{F}_{\sigma}=\frac{1}{2} q_{\sigma} \nabla\left\langle\mathbf{r}_{1} \cdot \mathbf{E}_{1}\right\rangle,
$$

where

$$
\begin{gathered}
\mathbf{r}_{1}=-\frac{q_{\sigma}}{m_{\sigma}}\left[\frac{\omega_{c \sigma} \hat{z} \times \dot{\mathbf{E}}_{1}}{\omega^{2}-\omega_{c \sigma}^{2}}+\frac{\hat{z} \times\left(\mathbf{E}_{1} \times \hat{z}\right)}{\omega^{2}-\omega_{c \sigma}^{2}}\right. \\
\left.+\frac{\left(\hat{z} \cdot \mathbf{E}_{1}\right) \hat{z}}{\omega^{2}}\right]
\end{gathered}
$$

is the first-order particle displacement due to the wave. Here the wave electric field is $\mathbf{E}_{1}=$ $\operatorname{Re}[\mathbf{E}(\mathbf{x}) \exp (-i \omega t)], q_{\sigma}, m_{\sigma}$, and $\omega_{c \sigma}$ are the charge, mass, and gyrofrequency of species $\sigma$, and \langle\rangle denotes time averaging over one wave period. Explicit evaluation of $\left\langle\mathbf{r}_{1} \cdot \mathbf{E}_{1}\right\rangle$ shows that the ponderomotive force is

$$
\begin{aligned}
\mathbf{F}_{\sigma}= & -\frac{q_{\sigma}^{2}}{4 m_{\sigma}} \nabla \\
& \times\left(\frac{\omega_{c \sigma} \hat{z} \cdot \dot{\mathbf{E}}_{\perp} \times \mathbf{E}_{\perp}^{*}}{\omega^{2}\left(\omega^{2}-\omega_{c \sigma}^{2}\right)}+\frac{\left|\mathbf{E}_{\perp}\right|^{2}}{\left(\omega^{2}-\omega_{c \sigma}^{2}\right)}+\frac{\left|E_{z}\right|^{2}}{\omega^{2}}\right),
\end{aligned}
$$

which is equivalent to Eq. (5) of Gurevich and Pitaevskii [13].

In order to determine the relative importance of the terms in $\mathbf{F}_{\sigma}$, consider the properties of inertial Alfvén waves in a cylindrical coordinate system $(r, \theta, z)$ (i.e., the natural coordinate system for resonance cones). Thus, the inertial Alfvén cones result from a superposition of modes of the form $\sim f(r) \exp \left(i k_{z} z-i \omega t\right)$. The parallel current $J_{z}$ for these waves is primarily due to field-aligned electron motion and is given by

$$
-i \omega \mu_{0} J_{z}=\frac{\omega_{p e}^{2}}{c^{2}} E_{z},
$$

while the perpendicular current is primarily due to ion polarization current and is given by

$$
\mu_{0} J_{r}=-\frac{i \omega}{v_{A}^{2}} E_{r} .
$$

These currents are related to the wave magnetic field via Ampere's law

$$
\mu_{0} J_{z}=\frac{1}{r} \frac{\partial}{\partial r}\left(r B_{\theta}\right), \quad \mu_{0} J_{r}=-i k_{z} B_{\theta},
$$

which, together with the $\theta$ component of Faraday's law,

$$
i k_{z} E_{r}-\frac{\partial E_{z}}{\partial r}=i \omega B_{\theta},
$$

provide the complete set of equations for cylindrically symmetric inertial Alfvén modes. These equations show that

$$
E_{r}=\frac{i k_{z} v_{A}^{2}}{\omega^{2}-k_{z}^{2} v_{A}^{2}} \frac{\partial E_{z}}{\partial r} .
$$

Combining Eqs. (4)-(8) gives an equation in $E_{z}$ with solution

$$
E_{z}=\bar{E}_{z} J_{0}\left(k_{\perp} r\right),
$$

where $\bar{E}_{z}$ is a constant and $k_{\perp}^{2}=\left(k_{z}^{2} v_{A}^{2} / \omega^{2}-1\right) \omega_{p e}^{2} / c^{2}$. Using Eq. (8) gives

$$
E_{r}=i \bar{E}_{z} \frac{k_{z}}{k_{\perp}} \frac{v_{A}^{2}}{c^{2}} \frac{\omega_{p e}^{2}}{\omega^{2}} J_{1}\left(k_{\perp} r\right),
$$

and $B_{\theta}, J_{r}$, and $J_{z}$ can similarly be obtained using Eqs. (4), (5), and (6).

We now examine the relative importance of terms in $\mathbf{F}_{\sigma}$ for the inertial Alfvén frequency regime where $\omega \ll \omega_{c i}, \omega_{c e}$. As shown in Eq. (6) of Ref. [11] the general form of Eq. (8) indicates that $\mathbf{E}_{\perp}$ is the gradient of a scaler so that $\hat{z} \cdot \dot{\mathbf{E}}_{\perp} \times \mathbf{E}_{\perp}^{*}$ is identically zero; i.e., the perpendicular electric field of inertial Alfvén waves cannot be circularly polarized, and the first term in Eq. (3) vanishes. Although, strictly speaking, the calculation of $\left|E_{z}\right|^{2}$ and $\left|E_{r}\right|^{2}$ requires superposition of the Fourier modes, we can compare the relative magnitudes of $\left|E_{z}\right|^{2}$ and $\left|E_{r}\right|^{2}$ by considering the behavior of a typical Fourier mode. $E_{z}$ is at a maximum at $r=0$ at which point $E_{r}$ vanishes; thus, at $r=0$ the dominant ponderomotive term is the $\mathbf{F}_{e}$ term involving $\left|E_{z}\right|^{2} / \omega^{2}$. In contrast, $E_{r}$ reaches a maximum when $k_{\perp} r \approx 2$ at which point $E_{z}$ vanishes, and at this point the dominant ponderomotive term is the ion term involving $\left|E_{r}\right|^{2} /\left(\omega^{2}-\omega_{c i}^{2}\right) \approx-\left|E_{r}\right|^{2} / \omega_{c i}^{2}$. The electron ponderomotive force is therefore $\mathbf{F}_{e} \simeq-\left(n_{e} q_{e}^{2} / 4 m_{e}\right) \nabla\left(\left|E_{z}\right|^{2} / \omega^{2}\right)$ and acts to expel electrons from the region $r \approx 0$ where $J_{0}^{2}(\kappa r)$ is at a maximum; the ion ponderomotive force is $\mathbf{F}_{i} \simeq+\left(n_{i} q_{i}^{2} / 4 m_{e}\right) \nabla\left(\left|E_{r}\right|^{2} / \omega_{g m}^{2}\right)$ and acts to attract ions to the region $k_{\perp} r \approx 2$ where $J_{1}^{2}\left(k_{\perp} r\right)$ is at a maximum. Comparing $\left|E_{r}\right|^{2} / \omega_{g m}^{2}$ to $\left|E_{z}\right|^{2} / \omega^{2}$ shows that the ion ponderomotive force is about an order of magnitude weaker than the electron ponderomotive force. Thus, the combined effect of ponderomotive forces should be to form an $\mathbf{F}_{e}$-induced density cavity bounded by a small, $\mathbf{F}_{i}$-induced density ridge.

The parallel component of the equilibrium fluid equations of motion are

$$
\begin{aligned}
& 0=-\frac{n_{e} q_{e}^{2}}{4 m_{e}} \frac{\partial}{\partial s}\left(\frac{\left|E_{z}\right|^{2}}{\omega^{2}}\right)-n_{e} q_{e} \frac{\partial \phi}{\partial s}-\frac{\partial}{\partial s}\left(n_{e} \kappa T_{e}\right), \\
& 0=+\frac{n_{i} q_{i}^{2}}{4 m_{e}} \frac{\partial}{\partial s}\left(\frac{\left|E_{r}\right|^{2}}{\omega_{g m}^{2}}\right)-n_{i} q_{i} \frac{\partial \phi}{\partial s}-\frac{\partial}{\partial s}\left(n_{i} \kappa T_{i}\right),
\end{aligned}
$$

where $\phi$ is an ambipolar potential [13] to be determined and $s$ is the distance along a field line. These equilibrium equations apply to the superposition of the Fourier modes; this superposition results in a $z$-dependent structure for 
$\left|E_{z}\right|^{2}$ and $\left|E_{r}\right|^{2}$. Integrating these equilibrium equations along the magnetic field gives

$$
\begin{aligned}
& n_{e}=n_{e 0} \exp \left[-\left(e^{2}\left|E_{z}\right|^{2} / 4 m_{e} \omega^{2}-e \phi\right) / \kappa T_{e}\right], \\
& n_{i}=n_{i 0} \exp \left[\left(e^{2}\left|E_{r}\right|^{2} / 4 m_{e} \omega_{g m}^{2}-e \phi\right) / \kappa T_{i}\right],
\end{aligned}
$$

using $q_{e}=-e$ and $q_{i}=+e$. In order to maintain quasineutrality, the ambipolar potential must be

$$
\phi=\frac{e}{4 m_{e}\left(T_{e}+T_{i}\right)}\left[\frac{\left|E_{z}\right|^{2} T_{i}}{\omega^{2}}+\frac{\left|E_{r}\right|^{2} T_{e}}{\omega_{g m}^{2}}\right],
$$

and the electron density satisfying quasineutrality will be

$$
n=n_{e 0} \exp \left[-\frac{e^{2}}{4 m_{e} \kappa\left(T_{e}+T_{i}\right)}\left(\frac{\left|E_{z}\right|^{2}}{\omega^{2}}-\frac{\left|E_{r}\right|^{2}}{\omega_{g m}^{2}}\right)\right] .
$$

From Eq. (4) we see that $\left|E_{z} / \omega\right|=c^{2} \mu_{0}\left|J_{z}\right| / \omega_{p e}^{2}$ so near $r=0$ the ambipolar potential will be

$$
\phi=\frac{m_{e}\left|J_{z}\right|^{2}}{4 n^{2} e^{3}\left(1+T_{e} / T_{i}\right)},
$$

while the electron density near $r=0$ will be given by the transcendental relation

$$
n_{e} / n_{e 0}=\exp \left[-\lambda n_{e 0}^{2} / n_{e}^{2}\right]
$$

where

$$
\lambda=m_{e}\left|J_{z}\right|^{2} / 4 n_{e 0}^{2} e^{2} \kappa\left(T_{i}+T_{e}\right) ;
$$

this transcendental relation is our main result. An interesting feature of this result is that low $\beta$ plasmas are particularly susceptible to ponderomotive forces, since low $\beta$ plasmas have low pressures and yet, because of the large equilibrium magnetic field, can support intense wave magnetic fields that are still small relative to the equilibrium magnetic field.

For small $\lambda$, the density perturbation $\Delta n_{e}=n_{e}-n_{e 0}$ can be approximated as

$$
\frac{\Delta n_{e}}{n_{e 0}}=\frac{n_{e}-n_{e 0}}{n_{e 0}} \approx-\lambda,
$$

while for large $\lambda$, Eq. (16) must be solved exactly. There are two roots $n_{e} / n_{e 0}$ for each $\lambda$; this can be seen by rewriting Eq. (16) as $\lambda=\left(n_{e} / n_{e 0}\right)^{2} \ln \left(n_{e 0} / n_{e}\right)$, and it is clear that both $n_{e} \rightarrow 0$ (i.e., complete density depletion) and $n_{e} \rightarrow n_{e 0}$ (i.e., no density depletion) give $\lambda \rightarrow 0$ (i.e., no parallel current). The two roots of Eq. (16) coalesce when $n_{e} / n_{e 0}=\exp (-1 / 2)=0.6$ which occurs when $\lambda=0.18$; this point of coalescence gives a maximum possible density depletion of 0.4 , somewhat less than the largest observed depletions. The ambipolar potential corresponding to maximum depletion is $\phi \simeq \kappa T_{i} / 5 e$.

Since the wave frequency is very low $(\sim 1 \mathrm{~Hz})$, the apparent time dependence $B_{\perp}(t)$ in the spacecraft frame comes almost entirely from spacecraft motion, i.e., $d B_{\perp} / d t \approx v_{s} \nabla B_{\perp}$. Thus, from Ampere's law the field-aligned current density is

$$
J_{z} \approx \frac{1}{\mu_{0} v_{s}} \frac{d B_{\perp}}{d t} .
$$

Figure 2(a) shows a plot of $\lambda$ evaluated using Eq. (17) assuming $T_{e}=T_{i}=1 \mathrm{eV}$, and $n_{e 0}=7.5 \times 10^{3} \mathrm{~cm}^{-3}$; also, $J_{z}$ was calculated by applying Eq. (19) to the data in Fig. 1(a). It is seen that $\lambda$ has very sharp peaks. Equation (16) predicts that there should be sharp density cavities at the location of these $\lambda$ peaks. Figure 2(b) replots the density shown in Fig. 1(b) with vertical dashed lines superimposed to indicate the location of the $\lambda$ peaks. There is an obvious correlation between the locations of the density cavities and the locations of the $\lambda$ peaks confirming the prediction that ponderomotive force due to parallel electric fields leads to density depletion. The moderate quantitative discrepancies could be explained by instrumental effects in the electron density measurement by Langmuir probe.

It is worth comparing $E_{r}^{\mathrm{amb}}$, the perpendicular electric field due to gradient of the ponderomotive potential, to

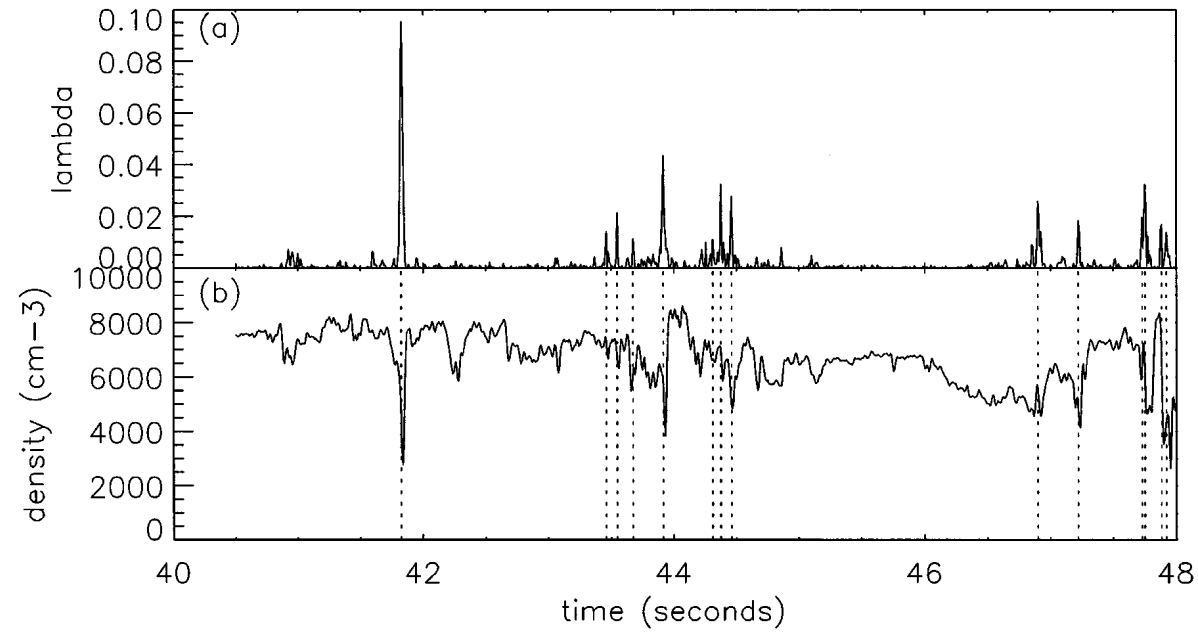

FIG. 2. (a) $\lambda$ calculated using measured $J_{\|}$obtained by using Eq. (19) on data in Fig. 1(a). (b) Density [same as in Fig. 1(b)], dotted vertical lines show times when $\lambda$ is at a local maximum. 
$E_{r}^{\text {wave }}$, the perpendicular wave electric field. We estimate the order of magnitude of the perpendicular wave field by combining Eqs. (4) and (10) to obtain for $k_{\perp} r \sim \mathcal{O}$ (1) and $\omega_{p e} r / c \sim \mathcal{O}(1)$

$$
\left|E_{r}^{\mathrm{wave}}\right| \sim v_{A} \frac{c}{\omega_{p e}} \mu_{0}\left|J_{z}\right|=\frac{B_{0}}{n e} \sqrt{\frac{m_{e}}{m_{i}}}\left|J_{z}\right| .
$$

Using $B_{0}=2.6 \times 10^{-5} \mathrm{~T}, n \sim(3-7) \times 10^{9} \mathrm{~m}^{-3}, J_{z} \sim$ $1.5 \times 10^{-4} \mathrm{~A} \mathrm{~m}^{-2}$, gives $E_{r}^{\text {wave }} \sim 100-200 \mathrm{mV} / \mathrm{m}$ for a hydrogen plasma and $E_{r}^{\text {wave }} \sim 20-50 \mathrm{mV} / \mathrm{m}$ for an oxygen plasma. These electric fields are in reasonable agreement with the observations. The wave potential is of the order of $E_{r}^{\text {wave }} c / \omega_{p e}$ and, since $c / \omega_{p e} \sim 10^{2} \mathrm{~m}$, is $5-20 \mathrm{~V}$ for hydrogen and $1-4 \mathrm{~V}$ for oxygen. In contrast, using Eq. (15) with $J_{z} \sim 1.5 \times 10^{-4} \mathrm{~A} \mathrm{~m}^{-2}$, $n \sim 3 \times 10^{9} \mathrm{~m}^{-3}, T_{e}=T_{i}$ gives the ambipolar potential to be $\phi \sim 0.01-0.1 \mathrm{~V}$. Thus, the ambipolar potential and field are much smaller than the wave potential and field. It is interesting that Eq. (4) gives $\left|E_{z}\right|=\omega \mu_{0}\left|J_{z}\right| c^{2} / \omega_{p e}^{2} \sim$ $10 \mu \mathrm{V} / \mathrm{m}$, an electric field much too small to be measured directly by electrostatic probes but nevertheless very important for the creation of significant density structures in space plasmas as we have demonstrated in this Letter.

The Freja project was financed by the Swedish National Space Board and the German Ministry for Science and
Technology. P. M. B. was supported in part by the National Science Foundation.

[1] For a detailed description of Freja instrumentation see Space Sci. Rev. 70, 405-602 (1994).

[2] D.-J. Wu, D.-Y. Wang, and C.-G. Fälthammar, Phys. Plasmas 2, 4476 (1995).

[3] D.-J. Wu, D. Y. Wang, and G. L. Huang, Phys. Plasmas 4, 611 (1997).

[4] C. E. Seyler, J.-E. Wahlund, and B. Holback, J. Geophys. Res. 100, 21453 (1995).

[5] P. K. Shukla and L. Stenflo, Phys. Scr. T60, 32 (1995).

[6] V. M. Chmyrev et al., Phys. Scr. 38, 841 (1988).

[7] K. Stasiewicz et al., J. Geophys. Res. 102, 2565 (1997).

[8] G. J. Morales and Y.C. Lee, Phys. Rev. Lett. 35, 930 (1975).

[9] G. G. Borg et al., Plasma Phys. Controlled Fusion 27, 1125 (1985).

[10] W. Gekelman, D. Leneman, J. Maggs, and S. Vincena, Phys. Plasmas 1, 3775 (1994).

[11] P. M. Bellan, Geophys. Res. Lett. 23, 1717 (1996).

[12] B. Bhattacharyya, T. Watanabe, and K. Nishikawa, J. Phys. Soc. Jpn. 59, 2776 (1990).

[13] A. V. Gurevich and L. P. Pitaevskii, Sov. Phys. JETP 18, 855 (1964). 